\title{
Enhance Derivative Design Considering Global Sensitivity of Design Parameters
}

\author{
Hyeong-UK Park ${ }^{1}$, Kamran Behdinan ${ }^{2}$, Joon Chung ${ }^{3}$, and Jae-Woo Lee ${ }^{4}$ \\ 1. Ph.D candidate, Department of Aerospace Eng., Ryerson University, 350 Victoria Street, Toronto, Ontario, M5B 2K3, Canada \\ 2. Professor, Department of Mechanical Eng., University of Toronto, 5 King's College Road, Toronto, Ontario, M5S 3G8, Canada \\ 3. Associate Professor, Department of Aerospace Eng., Ryerson University, 350 Victoria Street, Toronto, Ontario, M5B 2K3, Canada \\ 4. Professor, Aerospace Information Eng. Konkuk University, 1 Hwayang-dong, Gwangjin-gu, Seoul 143-701, South Korea
}

\begin{abstract}
An engineering product design considers derivatives to reduce the life cycle cost and to increase the efficiency on operation when it has new demands. The proposed design process in this study obtains derivative designs based on sensitivity of design variable. The efficiency and accuracy of the derivative design process can be enhanced by implementing global sensitivity analysis. Sensitivity analysis sensors the design variables accordingly and variables with low sensitivity for objective function can be neglected, since computational effort and time is not necessary for a design with less priority. In this research, e-FAST method code for global sensitivity analysis module was developed and implemented on Multidisciplinary Design Optimization (MDO) problem. The wing design was considered for MDO problem that used aerodynamics and structural disciplines. The global sensitivity analysis method was applied to reduce the number of design variables and Collaborative Optimization (CO) was used as MDO method. This research shows the efficiency of reduction of dimensionality of complex MDO problem by using global sensitivity analysis. In addition, this result shows important design variables for design requirement to student when they solving design problem.
\end{abstract}

Keyword : Multidisciplinary Design Optimization, Global Sensitivity Analysis, Extended Fourier Amplitude Sensitivity Test, Wing Design

\section{Introduction}

Many aircrafts have derivatives to satisfy various market requirements. Aircraft manufacturers develop new aircraft models as modifications or extensions of existing aircraft to meet new market demands while keeping the development time and cost to a minimum. However, the redesign of existing aircraft does require additional development resources and time. Therefore, a new design process that can reduce the cost and time for aircraft variation design is needed. In this research, a design process is studied which obtains global changes from local changes in aircraft design in order to develop aircraft derivatives efficiently.

In recent years, high fidelity tools and parallel computing are implemented for Multidisciplinary Design Optimization (MDO) to increase the accuracy of result since enhanced computing power. MDO of complex system as aircraft design has large number of design variables. A number of parameters is required to describe an aircraft depends on the level of maturity of the design and it can vary from tens to a few thousand. If design variables that have not significant effect on the optimization result, computational effort and time for these variables are not needed. Hence, identification of important factors on complex design problem is needed to reduce the dimensionality of MDO problem. Many sensitivity analysis methods are applied to engineering problems to identify the important parameters in design optimization problems [1, 6, 7]. Global sensitivity analysis offers a comprehensive approach to the model analysis and evaluates the effect of a factor while all other factors also are varied [1, 2]. Sobol' suggested the method of global sensitivity indices (1990) [3]. Then further developed by Saltelli and Sobol' (1995) [4]. Homma and Saltelli (1996) [5] are developed one of the most efficient and popular global sensitivity analysis techniques

In this research, the necessary design variables and parameters on MDO problem were identified by a global sensitivity analysis method. The design variables that had low sensitivity for objective were fixed as initial value. This work reduced the number of design variables then it reduced the calculation time and cost for design optimization. From the sensitivity information, student can make an objective observation which design variables are necessary to accomplish the requirement. The proposed method was applied to wing box design problem to show its efficiency. 


\section{Derivative design process and methods}

\subsection{Derivative design process}

Derivative designs for engineering products provide a cost effective design approach [8]. Many researchers have studied aircraft derivatives design using several different approaches. Researches of R. Hibma (1981), R. Birrenbach et al. (2000), R.B. Brown et al. (2001), and D. Kumar et al. (2006) identified the most important criteria for derivative designs using market requirement analysis. These researches considered altering constraints for the design problem [9, 10, 11, 12, 13]. J.D. Yearsley et al. (2008) researched on the generation of a Pareto frontier in order to identify the candidate product family members [14, 15]. T.W. Simpson et al. (2002, 2006, 2007) employed derivative design using a Multi-Objective Genetic Algorithm (MOGA). The product platform and its corresponding family of products were simultaneously optimized using a genetic algorithm while considering levels of platform [16, 17, 18]. Furthermore, J. Allison et al. (2006), and D. Lim and D. N. Mavris (2007) proposed evolutionary design method in order to design aircraft family [19, 20].

The previous researches of derivative design considered the whole set of design variables simultaneously to design the baseline and its derivatives together. In addition, these researches considered derivatives on conceptual design stage by assuming expected changes in product requirements. From the assumptions, various operation scenarios were generated and various types of derivative were developed to satisfy requirements. However, these assumptions may not accurately reflect future market changes. Furthermore, frozen requirements for design need to be redefined for derivatives when new requirements emerge.

The proposed design process obtains derivative designs based on requirements change and considers design variable selections. The sensitivity analysis result indicates the most necessary design variables that need to be altered to satisfy the new requirements. This information is utilized to select the most necessary design variables. It is used to reduce the scope of the derivative design optimization problem.

\subsection{Global Sensitivity Analysis (GSA)}

This research implements the e-FAST (Extended Fourier Amplitude Sensitivity Test) method for the global sensitivity analysis method [3]. The e-FAST is based on the original FAST (Fourier Amplitude Sensitivity Test) method [5, 21, 22]. The e-FAST method computes the main effect contribution of each input factor to the variance of the output. The sampling procedure implemented in e-FAST defines a sinusoidal function of a particular frequency for each input parameter. The choice of sinusoidal function depends on the distribution of desired parameter values. The frequencies assigned to parameters must meet several criteria so that they can be distinguished within Fourier analysis. Due to the symmetry properties of trigonometric functions, the sinusoidal function will eventually repeat the same samples and re-sampling scheme is implemented to avoid this inefficiency. The primary advantage of e-FAST method over the original FAST is the ability to calculate both the first-order sensitivity and total-order sensitivity of each input parameter. While original FAST method partitions variance to each parameter, e-FAST method partitions variance into two categories: variance due to the parameter of interest and variance due to all other parameters. It is robust at low sample size and computational efficient [4]. In this research, e-FAST module was developed based on Visual FORTRAN.

\subsection{Collaborative optimization method}

The Collaborative Optimization (CO) method introduces a decomposed and decentralized bi-level optimization method. A system level optimization is responsible for providing target values for global design variables $z$ and system responses $y$. A local disciplinary level optimization assures that the discrepancies between disciplines vanish by enforcing compatibility constraints. It is modeled to minimize the interdisciplinary discrepancies while satisfying specific local constraints. The CO formulation can be stated at the system level as [29]: 


$$
\begin{aligned}
& \min . f\left(z_{S L}, y_{S L}\right) \\
& \text { subject to } J_{i}\left(z_{S L}, z_{i}^{*}, y_{S L}, y_{i}^{*}\left(x_{i}^{*}, y_{j}, z_{i}^{*}\right)\right)=0, \\
& \qquad j=1,2, \cdots, n, \quad j \neq i
\end{aligned}
$$

where, $J$ is the compatibility constraints, one for each discipline, and $z^{*}, y^{*}$ and $x^{*}$ are the optimal disciplinary optimization level results. The subscript $S L$ means system level. The $i^{\text {th }}$ disciplinary level optimization problem is formulated as:

$$
\begin{aligned}
& \text { min. } J_{i}=\sum\left(z_{S L}-z_{i}\right)^{2}+\sum\left(y_{S L}-y_{i}\right)^{2} \\
& \text { subject to } g_{i}\left(x_{i}, z_{i}, y_{i}\left(x_{i}, y_{j}, z_{i}\right)\right) \leq 0
\end{aligned}
$$

where, $g$ is the $i^{\text {th }}$ disciplinary constraint.

\section{Multidisciplinary wing design using GSA}

The proposed method was applied to the design optimization of a light jet aircraft wing design. Wing design problem evaluated accuracy and efficiency of the proposed method. The optimization results were compared when it changes the number of design variables from sensitivity analysis result. A surrogate model was developed from samples of Finite Element Method (FEM) analysis. The RSM was used for estimating the maximum stress and weight of the wing box [30].

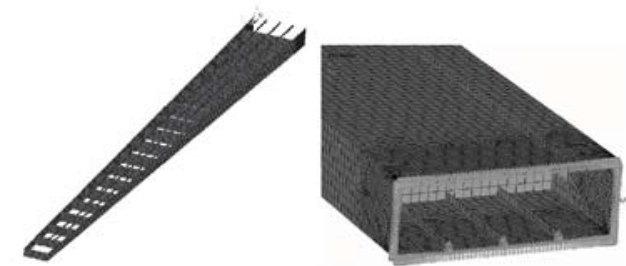

Figure 1. Wing box FEM model [31]

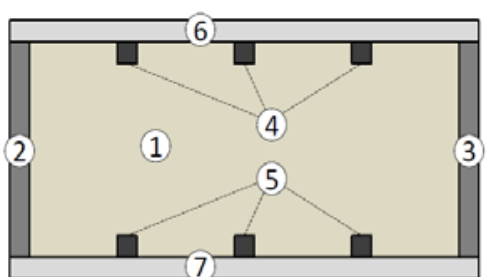

Figure 2. Wing box shape and design variables [31]

Table 1. Design variables for wing box and its range [31]

\begin{tabular}{c|c|c|c|c|c}
\hline Member & $\begin{array}{c}\text { Design } \\
\text { variable }\end{array}$ & Unit & Initial value & Lower limit & Upper limit \\
\hline Rib 1 19 & $x_{1}$ & in & 0.1379 & 0.0787 & 0.1969 \\
Front spar & $x_{2}$ & in & 0.7874 & 0.3937 & 1.1811 \\
Rear spar & $x_{3}$ & in & 0.7874 & 0.3937 & 1.1811 \\
Upper stringer 1 3 & $x_{4}$ & in & 0.2362 & 0.0787 & 0.3937 \\
Lower stringer 1 3 & $x_{5}$ & in & 0.2362 & 0.0787 & 0.3937 \\
Upper skin & $x_{6}$ & in & 0.8859 & 0.5906 & 1.1811 \\
Lower skin & $x_{7}$ & in & 0.8859 & 0.5906 & 1.1811 \\
Span length $(b)$ & $x_{8}$ & $f t$ & 31 & 26.0 & 36.0 \\
Reference wing area $(S)$ & $x_{9}$ & $f t^{2}$ & 113.25 & 96.50 & 130.0 \\
\hline
\end{tabular}

A parameterized finite-element model of a generic light business jet wing box was developed on ANSYS and is shown in Figure 1. A wing box model was automatically constructed including leading and trailing edge spars, upper and lower skins, as well as stringers and ribs by using developed a MATLAB function for ANSYS mesh generate. The MATLAB function derived the random cases of parameters to generate meshes on ANSYS. ANSYS developed meshes and analysis Von Mises stress and weigh of each case from MATLAB function. In this research, 148 cases were analyzed and RSM of wing weight and Von Mises stress were derived. The FEM 
model consisted of 29 member attributes representing the thicknesses of the primary structural members - 19 ribs, the front and rear spar, 6 stringers, the upper and lower skin, span, and wing reference area. The dimensionality was reduced by linking attributes to 9 design variables as shown in Figure 2 and Table 1. The sweep angle, taper ratio, and airfoil were fixed as constants.

\subsection{Design formulation}

The aircraft concept was considered similar in size and performance to light jet aircraft such as the Cessna Mustang and the D-Jet [32, 33]. In this research, higher $L / D$ was considered while minimizing weight of wing. The major design parameters for wing design are shown in table 2. The maximum Von Mises stress was constrained to be below $360 \mathrm{MPa}$, corresponding to the yield strength of aluminum 7075 with a safety margin of 1.5 as required by airworthiness standards. The objective of optimization was to maximize the wing lift-to-drag ratio at a cruise speed of $400 \mathrm{kts}$ and an altitude of 35,000 $\mathrm{ft}$ while the body contribution to the lift-to-drag ratio was neglected. The FEM analysis was replaced by the RSM.

Table 2. Design parameters for wing design

\begin{tabular}{c|c}
\hline Design parameter & Given value \\
\hline Gross weight of aircraft & $11,464.04 \mathrm{lb}$ \\
Wing stored fuel capacity & $2,645.55 \mathrm{lb}$ \\
Wing weight budget & $970.03 \mathrm{lb}$ \\
Max. von Mises stress & $360 \mathrm{MPa}$ \\
Cruise speed & $400 \mathrm{kts}$ \\
Cruising altitude & $35,000 \mathrm{ft}$ \\
\hline
\end{tabular}

The MDO problem was formulated with two disciplines: an aerodynamics solver using panel method and a structures solver consisting of the RSM. The RSM was generated from a database of sampled finite-element solutions in the design space. The random cases of wing box structure analysis were calculated on FEM model and these results were used to develop RSM model. The developed model had $R_{\text {adj }}^{2}=0.98$ and it showed the accuracy of the approximation model.

Table 3 shows the sensitivity indices for wing box weight that objective of structure module. Due to this result, seven design variables were selected and two design variables $\left(x_{3}, x_{4}\right)$ were fixed as initial value. The first order sensitivity index represents the fraction of model output variance that is explained by the input variation of a given parameter. On the other hand, the total sensitivity index shows the factor's main effect and all the interaction terms of that factor. Total sensitivity index is defined as the sum of all the sensitivity indices involving that factor. The MDO formulation was generated using seven design variables. Figure 4 shows the architecture of this $\mathrm{CO}$ formulation.

Table 3. Sensitivity indices of design variables

\begin{tabular}{c|r|r|r}
\hline $\begin{array}{c}\text { Design } \\
\text { variables }\end{array}$ & $\begin{array}{c}1^{\text {st }} \text { order } \\
\text { Sensitivity } \\
\text { Index }\end{array}$ & $\begin{array}{c}\text { Total } \\
\text { Sensitivity } \\
\text { Index }\end{array}$ & Rank \\
\hline$x_{1}$ & 0.0030 & 0.04 & 5 \\
$x_{2}$ & 0.0025 & 0.0339 & 6 \\
$x_{3}$ & 0.0013 & 0.0172 & 8 \\
$x_{4}$ & 0.0012 & 0.0159 & 9 \\
$x_{5}$ & 0.0045 & 0.0600 & 4 \\
$x_{6}$ & 0.0023 & 0.0306 & 7 \\
$x_{7}$ & 0.0134 & 0.1798 & 3 \\
$x_{8}$ & 0.7087 & 9.4987 & 1 \\
$x_{9}$ & 0.2631 & 3.5269 & 2 \\
\hline \multicolumn{4}{|c}{4}
\end{tabular}




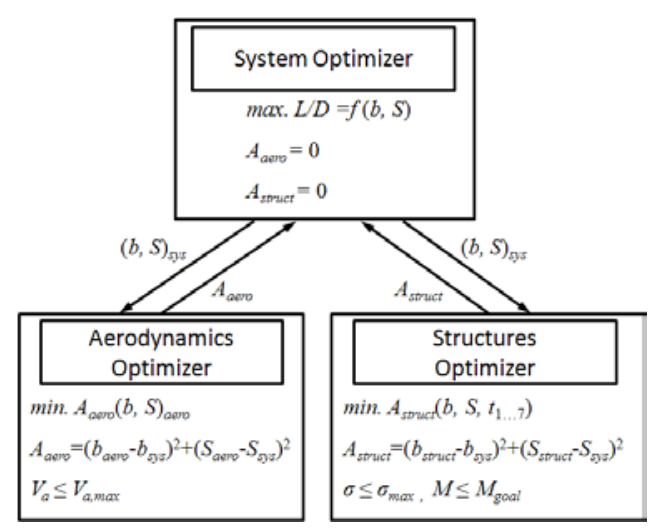

Figure 4. $\mathrm{CO}$ architecture

The system objective function was maximize $L / D$. The $A_{\text {aero }}$ and $A_{\text {struct }}$ were auxiliary constraint of aerodynamics and structures discipline respectively. The $t$ was structure thickness value, $M$ was mass of wing, and $V_{a}$ was approaching speed. Table 4 and Table 5 show the constraints and formulations of each method for structures discipline respectively. This research used the approach speed for constraints of aerodynamic discipline. Other aircraft characteristics for aerodynamic efficiency as range and endurance need parameters from performance and thrust discipline but in this problem, these disciplines were not considered in this problem. On the other hand, approach speed can be derived from given parameters in this MDO problem and it can evaluate the system objective function.

Table 4. Constraints for wing box conceptual design

\begin{tabular}{c|c|c}
\hline Constraints & Symbol & Value \\
\hline Maximum stress & $\sigma$ & $\leq 360 \mathrm{MPa}$ \\
Mass & $M$ & $\leq 970.03 \mathrm{lb}$ \\
Approach speed & $V_{a}$ & $\leq 120 \mathrm{kts}$ \\
\hline
\end{tabular}

Table 5. Comparison of design result

\begin{tabular}{c|r|r}
\hline $\begin{array}{c}\text { Design } \\
\text { variables }\end{array}$ & $\begin{array}{c}\text { Case 1 } \\
\text { (Deterministic } \\
\text { optimization) }\end{array}$ & $\begin{array}{c}\text { Case 2 } \\
\text { (Selected design } \\
\text { variables) }\end{array}$ \\
\hline$x_{1}$ & 0.0906 & 0.0906 \\
$x_{2}$ & 0.7126 & 0.7047 \\
$x_{3}$ & 0.5984 & - \\
$x_{4}$ & 0.1850 & - \\
$x_{5}$ & 0.2362 & 0.2362 \\
$x_{6}$ & 0.7244 & 0.8858 \\
$x_{7}$ & 0.5866 & 0.8858 \\
$x_{8}$ & 33.7927 & 33.7927 \\
$x_{9}$ & 114.0975 & 114.0975 \\
\hline$L / D$ & 31.9 & 30.8 \\
Error & - & $3.45 \%$ \\
\hline Number of & 158 & 104 \\
iteration & - & $33.55 \%$ \\
Improvement & - &
\end{tabular}

\subsection{Discussion}

The fixed design variables were thickness of rear spar and upper stinger that had less importance on wing weight. From this result, case 2, which reduced the number of design variable showed $3.45 \%$ error and it 
reduced $33.55 \%$ of the number of iteration. It means that the reduced number of design variable using e-FAST method can be applied to conceptual design and the proposed derivative design process can be useful when it considers the selection of design variables for new demands on wing structure weight.

\section{Conclusion}

In this research, the enhanced derivative design method was studied. The global sensitivity analysis method identifies the most important design variables and which design variables can be omitted to accomplish the design objective. The reduction of number of design variables decreases the calculation time for optimization. The accuracy of developed e-FAST module was evaluated by the wing design optimization problem. It showed small error and less iteration number when reduced number of design variables was applied.

The sensitivity analysis result can be used to not only RSM but also FEM analysis. The number of design variable has highly effect on calculation time of high fidelity analysis tool as FEM and CFD. The proposed method is useful on conceptual design level when it uses high fidelity analysis tool. Additional process as global sensitivity analysis can increase total calculation time. Though, the result of sensitivity analysis can be used on derivative design when the requirement for objective function is changed again and it can reduce the time for case study for student when they solve engineering problem and it shows objective criteria for important design variables for design goal.

\section{References}

1. A. Saltelli, S. Tarantola, F. Campolongo, and M. Ratto, Sensitivity Analysis in Practice. A Guide to Assessing Scientific Models, John Wiley \& Sons publishers, Probability and Statistics series, 2004.

2. A. Saltelli, K. Chan and M. Scott, Eds., Handbook of sensitivity analysis, John Wiley \& Sons publishers, Probability and Statistics series, 2000.

3. I. Sobol', Sensitivity estimates for nonlinear mathematical models. Matem Modelirovanie, vol. 2, no. 1, pp.112-118, 1990 [in Russian]. English translation : Math Model Comput Exp, vol. 1, no. 4, pp. 407414, 1993.

4. A. Saltelli, I. Sobol'. About the use of rank transformation in sensitivity analysis of model output. Reliability Engineering \& System Safety, vol. 50, issue 3, pp. 225-239, 1995.

5. H.C. Frey, S.R. Patil, Identification and review of sensitivity analysis methods, Risk Analysis, vol. 22, issue 3, pp.553-578. Jun. 2002.

6. C. Xu, G. Gertner, Extending a global sensitivity analysis technique to models with correlated parameters, Computational Statistics \& Data Analysis, vol. 51, pp. 5579 - 5590, 2007.

7. B.Y. Kim, C.M. Jeong, S.W. Kim, M.W. Suh3, A study to maximize the crash energy absorption efficiency within the limits of crash space, Journal of Mechanical Science and Technology, Vol.26, No.4, pp. 1073 1078, 2012.

8. R.E. Perez, J. Chung, and K. Behdinan. Aircraft conceptual design using genetic algorithms. AIAA-2000-4938, AIAA/USAF/NASA/ISSMO Symposium on Multidisciplinary Analysis and Optimization, 8th, Long Beach, CA, Sept. 6-8, 2000.

9. R. Hibma, D. Wegner, The Evolution of a Strategic Bomber, AlAA 1981 Annual Meeting and Technical Display, Long Beach, California, May 12-14, 1981.

10. R.H. Fulford, Airplane Criteria Process, World Aviation Congress \& Exposition, Anaheim, CA, USA, Oct. 1997.

11. R. Birrenbach, Regional Aircraft Family Design, 2000 World Aviation Conference, San Diego, CA, Oct. 10-12, 2000.

12. R.B. Brown, J.M. Swihart, A New Family of Passenger Friendly Commercial Air Transports, 39th 
AIAA Aerospace Sciences, Meeting \& Exhibit, Reno. Jan. 8-11, 2001.

13. D. Kumar, W. Chen, T.W. Simpson, A Market-Driven Approach to the Design of Platform-Based Product Families, 11th AIAA/ISSMO Multidisciplinary Analysis and Optimization Conference, Portsmouth, Virginia, Sept. 6-8, 2006.

14. J.D. Yearsley, C.A. Mattson, Product Family Design using a Smart Pareto Filter, 46th AIAA Aerospace Sciences Meeting and Exhibit, Reno, Nevada, Jan. 7-10, 2008.

15. J.D. Yearsley, C.A. Mattson, Interactive Design of Combined Scale-based and Module-based Product Family Platforms, 12th AIAA/ISSMO Multidisciplinary Analysis and Optimization Conference, Victoria, British Columbia Canada, Sept. 10-12, 2008.

16. T.W. Simpson, B. D’Souza, Assessing Variable Levels of Platform Commonality Within A Product Family Using A Multiobjective Genetic Algorithm, 9th AIAA/ISSMO Symposium on Multidisciplinary Analysis and Optimization, Atlanta, Georgia, Sept. 4-6, 2002.

17. S. Valliyappan, T.W. Simpson, Exploring Visualization Strategies to Support Product Family Design Optimization, 11th AIAA/ISSMO Multidisciplinary Analysis and Optimization Conference, Portsmouth, Virginia, Sept. 6-8, 2006.

18. A. Khajavirad, J.J. Michalek, T.W. Simpson, A Decomposed Genetic Algorithm for Solving the Joint Product Family Optimization Problem, 48th AIAA/ASME/ASCE/AHS/ASC Structures, Structural Dynamics, and Materials Conference, Honolulu, Hawaii, Apr. 23-26, 2007.

19. D. Lim, D.N. Mavris. An Approach to Evolutionary Aircraft Design for Growth Potential, 7th AIAA Aviation Technology, Integration and Operations Conference (ATIO), Belfast, Northern Ireland, Sept. 18-20, 2007.

20. J. Allison, B. Roth, M. Kokkolaras, I. Kroo and P.Y. Papalambros, Aircraft Family Design Using Decomposition-based Methods, 11th AIAA/ISSMO Multidisciplinary Analysis and Optimization Conference, Portsmouth, Virginia, Sept. 6-8, 2006.

21. A. Saltelli, P. Annoni, How to avoid a perfunctory sensitivity analysis. Environmental Modeling \& Software, vol. 25, issue 12, pp. 1508-1517, Dec. 2010.

22. J. Yang, Convergence and uncertainty analyses in Monte-Carlo based sensitivity analysis. Environmental Modeling \& Software, vol. 26, issue 4, pp. 444-457, Apr. 2011.

23. S. Burge, A.A. Giunta, V. Balabanov, B. Grossman, W.H. Mason, R. Narducci, R.T. Haftka, L. Watson, A Coarse-Grained Parallel Variable-Complexity Multidisciplinary Optimization Paradigm, Intl. J. Supercomputing Applications and High Performance Computing, Vol. 10, No.4, pp. 269-299. Dec. 1996.

24. R. H. Myers, and D. C. Montgomery, Response Surface Methodology : Process and Product Optimization Using Designed Experiments, John Wiley \& Sons, New York, 1995.

25. J.W. Lee, B.Y. Min, Y.H. Byun, and S.J. Kim, Multi-Point Nose Shape Optimization of Space launcher Using Response Surface method, AIAA J. of Spacecraft and Rockets, Vol. 43, No. 1, Jan-Feb. 2006.

26. R.D. Braun, I. M. Kroo, Development and application of the collaborative optimization architecture in a multidisciplinary design environment, Technical Report, NASA Langley Technical Report Server, Aug. 14, 1995.

27. G.G. Wang and S. Shan, Review of meta modeling techniques in support of engineering design optimization, Journal of Mechanical Design, Vol. 129, pp. 370-381, Apr. 2007.

28. D. Neufeld, K. Behdinan, and J. Chung. Aircraft wing box optimization considering uncertainty in surrogate models. Structural and Multidisciplinary Optimization, Vol. 42, No. 5, pp. 745-753, Jul. 2010.

29. www.cessna.com

30. www.diamondaircraft.com 